\title{
Acromegaly and late-onset primary hyperparathyroidism in a female with a rare MEN1 gene variant of yet undetermined clinical significance (p.Val167Ala)
}

\author{
Magdalena Godlewska', Anna Bogusławska', Andrzej Nowak², Anna Skalniak1, Anna Sowa-Staszczak', \\ Aleksandra Gilis-Januszewska', Alicja Hubalewska-Dydejczyk ${ }^{T}$ \\ ${ }^{1}$ Department of Endocrinology, Jagiellonian University Medical College, Cracow, Poland \\ ${ }^{2}$ Department of Endocrinology, University Hospital in Cracow, Poland
}

Key words: acromegaly; MEN1; hyperparathyroidism; adrenal tumours; pituitary neuroendocrine tumour

We report a case of a female, born in 1952, diagnosed with a rare multiple endocrine neoplasia type 1 (MEN1) gene variant of uncertain clinical significance (p.Val167Ala) presenting with acromegaly, late-onset primary hyperparathyroidism (PHP), and bilateral adrenal tumours (Fig. 1).

The diagnosis of acromegaly due to pituitary macroadenoma was confirmed at the age of 45 years. The patient underwent a transsphenoidal resection of the pituitary tumour, with histopathological confirmation of adenoma chromophobe, which failed to achieve biochemical control of the disease. The patient refused consent to the second surgical approach or adjuvant radiotherapy. In 2002, pharmacological treatment with long-acting somatostatin analogue was introduced, resulting in successfully normalised insulin-like growth factor and growth hormone values.

Mild hypercalcaemia was discovered for the first time during endocrinological follow-up at the age of 56 years $(2.61 \mathrm{mmol} / \mathrm{L}$, normal range $2.15-2.55 \mathrm{mmol} / \mathrm{l})$, with elevated parathyroid hormone level (PTH) (87.4 $\mathrm{pg} / \mathrm{mL}$, normal range 14.90-56.90) and increased urine excretion of calcium $(7.01 \mathrm{mmol} / 24 \mathrm{~h}) .{ }^{99 \mathrm{~m} T c-M I B I}$ single-photon emission computed tomography/computed tomography (SPECT/CT) showed no typical focal accumulation of the tracer, only an increased uptake in the topography of the upper right parathyroid. Further follow-up of hypercalcaemia was planned.

The radiological image of the hypothalamic-pituitary area remained stable since medical treatment implementation: the last pituitary magnetic resonance imaging (MRI) in 2015 showed the remnants of the pituitary gland, less than $1 \mathrm{~mm}$ in diameter, at the bottom of the sella turcica and two hypointense masses enhancing heterogeneously after contrast administration: $11 \times 8 \times 13 \mathrm{~mm}$ in the right lateral part of the Turkish saddle and a similar $7 \times 4 \times 5 \mathrm{~mm}$ lesion on the left, in direct proximity to cavernous sinuses (Fig. 2). Also in 2015, a routine abdominal ultrasound revealed bilateral adrenal tumours. A low-density $25 \times 16 \times 21 \mathrm{~mm}$ tumour in the right adrenal gland and a similar $13 \mathrm{~mm}$ focal lesion in the left adrenal were confirmed in abdominal computed tomography (CT) (Fig. 3). Endocrinological evaluation detected no abnormal hormonal function of the adrenal tumours. Currently, at the age of 68 years, persistent elevation of PTH was confirmed, with normal calcium and phosphate concentrations and vitamin D level within the normal range. No focal lesions suggestive of enlarged parathyroid glands were revealed by neck ultrasound nor control ${ }^{99 \mathrm{~m} T c-M I B I}$ SPECT-CT. Densitometry showed osteopaenia of the lumbar vertebral column and proximal part of the arm, while nephrolithiasis and cholecystolithiasis were diagnosed in abdominal ultrasound. Insufficiency of the thyroid axis was diagnosed, and l-thyroxine substitution was implemented. Due to clinical manifestation of two characteristic features of MEN1 syndrome, genetic testing was performed. Sanger sequencing confirmed the MEN1 variant p.Val167Ala — a missense variant registered in NCBI dbSNP under the accession number rs748648909. In NCBI ClinVar, it was summarised to be of uncertain clinical significance (RCV000632131.2). So far, this variant has not been described in HGMD and UMD-MEN1 databases. The patient's family members have been invited for cascade screening. 


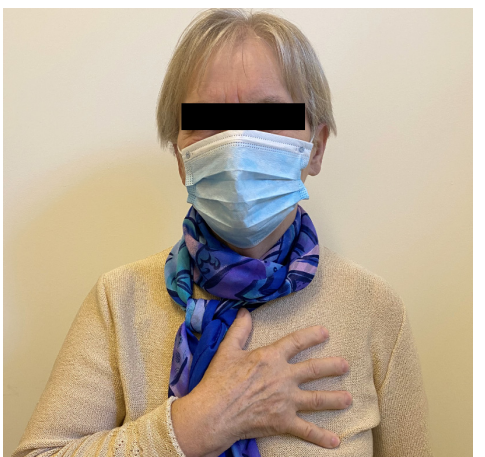

Figure 1. Clinical presentation of the patient - acral enlargement

Multiple neuroendocrine neoplasia type 1 is a rare genetic disorder with an autosomal dominant inheritance. The MEN1 gene, located on chromosome 11q13, encodes the protein menin, which directly regulates expression of the cyclin-dependent kinase-inhibiting (CKI) genes CDKN1b (encoding p27) and CDKN2C (encoding p18). Up to 1336 MEN1 gene sequence abnormalities were described within only 10 years following the identification of the gene [1]. In the case of clinical presentation of MEN1 but no detectable MEN1 mutation, MEN4 should be suspected. It is also referred to as MENX and is caused by heterozygous mutations of CDNK1B [2].

The phenotype-genotype correlation among MEN1 patients in terms of tumour localisation, age of onset, and clinical aggressiveness has not been established yet [2]. Usually the first manifestation of MEN1 syndrome is PHP, with almost $100 \%$ penetrance up to the age of 50 years. In our case, onset of PHP has been estimated at the age of 56 years. Even mild hypercalcaemia in patients presenting with MEN1-associated features should heighten awareness among health care practitioners [3]. Acromegaly related to MEN1 is diagnosed in about $10 \%$ of cases. MEN 1-associated somatotroph pituitary tumours are often macroadenomas with local aggressiveness and poor response to various treatment modalities [4]. Adrenal involvement among MEN1 patients should also heighten awareness, because those adrenal lesions tend to differ from sporadic incidentalomas. The relatively high frequency of primary hyperaldosteronism but also rare cases of pheochromocytoma (constituting pituitary adenoma/pheochromocytoma/paraganglioma association [3Pa]) should be considered during endocrinological evaluation [5].

Further follow-up of the patient and her family members should be carried out to confirm the spectrum and exact time of clinical presentation and, therefore, to determine the clinical significance of this rare MEN1 gene variant (p.Val167Ala).

\section{Authors' contributions}

M.G. and A.B. contributed equally to the manuscript.

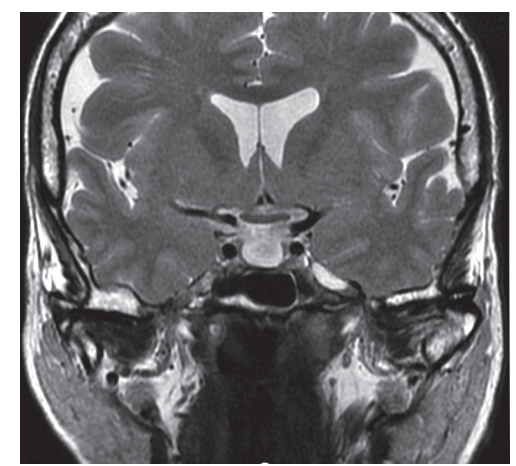

Figure 2. Coronal MRI image of the hypothalamic-pituitary area. Dominating radiological image of an empty sella. Remnants of the pituitary gland, less than $1 \mathrm{~mm}$ in diameter, at the bottom of the sella turcica and two hypointense masses $11 \times 8 \times 13 \mathrm{~mm}$ in the right lateral part of the Turkish saddle and a similar $7 \times 4$ $\times 5 \mathrm{~mm}$ lesion on the left, in direct proximity to cavernous sinuses

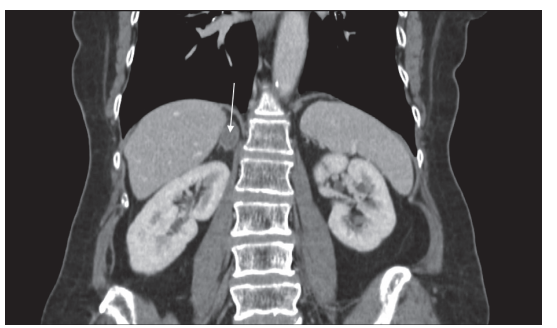

Figure 3. Coronal CT image of the abdomen. Tumour of the right adrenal gland (arrow)

\section{Funding}

This manuscript is a part of Jagiellonian University Medical College statutory research "Assessment of clinical, radiological, biochemical and genetic factors influencing the treatment efficacy in patients diagnosed with acromegaly" no. N41/DBS/00407. Ethics Comitee approval no. 1072.6120.72.2020.

\section{Conflict of interest}

None declared.

\section{References}

1. Lemos MC, Thakker RV. Multiple endocrine neoplasia type 1 (MEN1): analysis of 1336 mutations reported in the first decade following identification of the gene. Hum Mutat. 2008; 29(1): 22-32, doi: 10.1002/humu.20605, indexed in Pubmed: 17879353.

2. Thakker RV, Newey PJ, Walls GV, et al. Endocrine Society. Clinical practice guidelines for multiple endocrine neoplasia type 1 (MEN1). J Clin Endocrinol Metab. 2012; 97(9): 2990-3011, doi: 10.1210/jc.2012-1230, indexed in Pubmed: 22723327.

3. Nachtigall LB, Guarda FJ, Lines KE, et al. Clinical MEN-1 Among a Large Cohort of Patients With Acromegaly. J Clin Endocrinol Metab. 2020; 105(6), doi: 10.1210/clinem/dgaa142, indexed in Pubmed: 32311048.

4. Vergès B, Boureille F, Goudet $P$, et al. Pituitary disease in MEN type 1 (MEN1): data from the France-Belgium MEN1 multicenter study. J Clin Endocrinol Metab. 2002; 87(2): 457-465, doi: 10.1210/jcem.87.2.8145, indexed in Pubmed: 11836268.

5. Gatta-Cherifi B, Chabre O, Murat A, et al. Adrenal involvement in MEN1. Analysis of 715 cases from the Groupe d'etude des Tumeurs Endocrines database. Eur J Endocrinol. 2012; 166(2): 269-279, doi: 10.1530/EJE-11-0679, indexed in Pubmed: 22084155. 